\title{
Rate Dependence of Acoustic Emissions Generated during Shear of Simulated Fault Gouge
}

\author{
by Karen Mair, Chris Marone, and R. Paul Young
}

\begin{abstract}
Earthquake systems are commonly described using rate and state dependent fault models; however, the connection between rate and state friction parameters and specific microprocesses remains a challenge. We present new laboratory observations using modern ultrasonic techniques to reveal dynamic processes operating during frictional sliding. Granular layers were sheared under constant normal stress for a range of loading rates. During experiments, we monitored high-frequency acoustic emissions (AE) generated by grain fracture and friction using an array of piezoelectric transducers surrounding the layers. Complete waveforms and event information were collected for thousands of microearthquakes. Perturbations in imposed load point velocity $(V)$ produced a friction response consistent with previous work. For a given $V$, AE per sec decreased with accumulated slip, suggesting sensitivity to gouge layer evolution. Step increases in $V$ led to immediate and sustained increases in AE per sec; the converse was true for $V$ decreases. The positive rate dependence of $\mathrm{AE}$ per second is unsurprising because more slip is covered per unit time at higher $V$; however, AE per unit slip decreases with increasing $V$, indicating a deficit of acoustic activity at a faster slip rate. Assuming that AE result mainly from grain contact sliding, acoustic activity is proportional to the real area of contact between sliding particles. Our results qualitatively agree with previous experiments carried out on bare rock surfaces and support ideas that the frictional contact junction area is reduced at increased sliding velocity. We highlight a new way to visualize micromechanical contact processes important in frictional mechanics and highly relevant to earthquake physics.
\end{abstract}

\section{Introduction}

Earthquake systems are commonly described using rate and state dependent fault models (e.g., Dieterich, 1978, 1979; Dieterich et al., 1981; Marone, 1998; Scholz, 1998; Paterson and Wong, 2005). The parameters in these laws describe the microphysics of slip; however, a detailed understanding of the connection between friction parameters and specific microprocesses remains a challenge. We present new laboratory observations using state of the art ultrasonic techniques to gain insights into the dynamic processes operating during frictional sliding of granular materials. Understanding the behavior of sheared granular materials is important because mature fault zones exhibit significant accumulations of fault gouge and active faults, for example, San Andreas fault, are hosted at least at some depths in sedimentary or weakly consolidated rocks.

Acoustic emissions (AE) are high-frequency (kHz$\mathrm{MHz}$ ) elastic waves generated by the rapid release of energy associated with brittle fracture and sliding on a grain scale. They carry information regarding the location, intensity, and deformation mechanism of events occurring in a material under stress. AE resemble seismic waves generated by earthquakes but have lower amplitude and smaller source dimensions $(\sim \mathrm{mm})$. AE monitoring is a powerful tool in rock deformation because it gives real-time information on dynamic microprocesses associated with a specific macroscopic behavior. Importantly, it is a noninvasive method, hence it does not alter the process itself. AE has been successfully used in rock mechanics laboratory experiments to provide important insights into deformation processes operating during deformation of intact rock (e.g., Lockner, 1993) and sliding along precut "fault" surfaces (e.g., Weeks et al., 1978; Sammonds and Ohnaka, 1998, Yabe et al., 2003). Recent measurements of AE on precut surfaces (Yabe, 2002, Yabe et al., 2003) have revealed that AE activity shows a dependence on the imposed slip rate and a sensitivity to the accumulated slip.

In this article we present new observations of AE produced during shear deformation of granular layers. Our goal is to better understand the behavior of faults with a gouge. We show that AE activity is sensitive to accumulated slip and 
imposed slip rate, and we infer from our observations that the frictional contact area is inversely dependent on sliding velocity.

\section{Experimental Method}

\section{Friction Experiments}

Granular layers were sheared between rough forcing blocks in a servo controlled direct shear friction apparatus (Fig. 1). This geometry (Dieterich, 1978; Marone, 1998) and indeed this particular apparatus setup have been used extensively to investigate friction (e.g., Karner and Marone, 1998; Mair and Marone, 1999). Constant normal stress was applied and maintained on the side blocks; then the central block was driven at a specified load point velocity to initiate shear within the granular layers. Shear and normal stresses and displacements were continuously monitored during the tests. The resulting shear stress divided by normal stress yields the coefficient of sliding friction. Dilation and compaction of the layers indicates reorganization of particles associated with shear. Additional experimental details are gi- ven by Karner and Marone (1998) and by Mair and Marone (1999).

Direct shear experiments were conducted at a range of normal stresses $\left(\sigma_{n}=5-50 \mathrm{MPa}\right)$ and load point velocities ( $V=2-200 \mu \mathrm{m} / \mathrm{sec}$ ) under conditions of room temperature and humidity. These normal stress conditions correspond to regimes where previous work has indicated pervasive (50 $\mathrm{MPa}$ ) or minimal (5 MPa) comminution and grain size reduction during initial stages of shearing (Mair and Marone, 2000). With accumulated slip, grain comminution rate is significantly reduced (e.g., Sammis and Biegel, 1989), and grain dynamics in both "fracture" and "nonfracture" regimes are more strongly dominated by grain rolling (and perhaps grain boundary sliding). During our experiments, step change perturbations in load point velocity were imposed periodically, and the resulting variations in friction and acoustic activity were monitored. These velocity stepping experiments were carried out to investigate links between the rate dependence of friction and the associated acoustic activity produced by sheared granular materials. Experimental details are presented in Table 1 . (a)

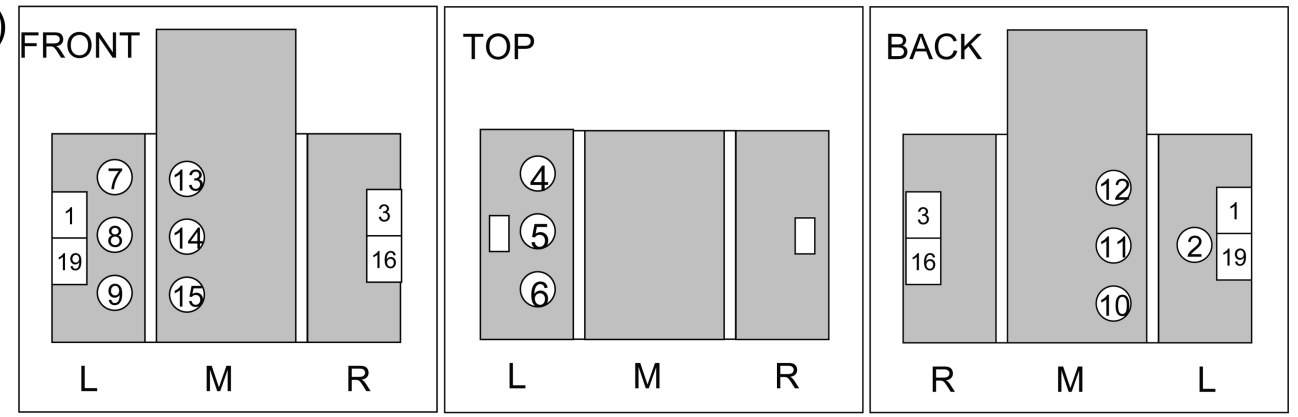

(b)

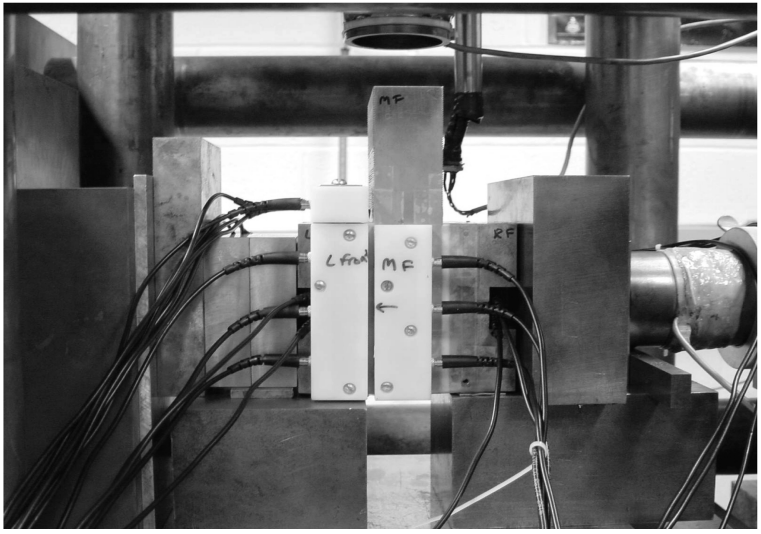

Figure 1. (a) Schematic diagram of double direct shear apparatus geometry showing transducer locations for the experiments. Steel forcing blocks, gray areas; gouge layers and sensors are numbered, light areas. Note some experiments had a reduced sensor array (see Table 1 for details). (b) Photograph of the transducer setup is shown. 
Table 1

Summary of Experiments Carried Out

\begin{tabular}{clclll}
\hline Experiment & Gouge/Blocks & \multicolumn{1}{c}{$\begin{array}{c}\text { Normal Stress } \\
\text { (MPa) }\end{array}$} & $\begin{array}{c}V \\
(\mu \mathrm{m} / \mathrm{sec})\end{array}$ & \multicolumn{1}{c}{ Sliding } & \multicolumn{1}{c}{ Ultrasonic } \\
\hline P163 & F110/Stl & $25 \mathrm{MPa}$ & $10-20-30$ & Stable & $\mathrm{AE} / \mathrm{Vel} / \mathrm{G}$ \\
P164 & SPH/Stl & $10 \mathrm{MPa}$ & & Stick slip & $\mathrm{AE} / \mathrm{G}$ \\
P166 & F110/Stl & $25 \mathrm{MPa}$ & $10-20-30$ & Stable & Vel \\
P167 & SPH/Stl & $40 \mathrm{MPa}$ & $6-10-30$ & Stick slip & $\mathrm{AE} / \mathrm{Vel} / \mathrm{G}$ \\
P169 & F110/granite & $10 \mathrm{MPa}(0-23 \mathrm{~mm})$ & $2-10-20-200$ & Stable & $\mathrm{AE} / \mathrm{Vel}$ \\
& & $15 \mathrm{MPa}(23-32 \mathrm{~mm})$ & & & \\
\hline
\end{tabular}

Terms used in this table are as follows: F110 - angular quartz sand (U.S. Silica Company); SPH-spherical glass beads (Mo-Sci Corporation); Stl-hardened steel forcing blocks (grooved faces); granite-Westerly granite forcing blocks (sandblasted faces); AE-AE waveforms recorded in triggered mode; $\mathrm{G}-\mathrm{AE}$ waveforms recorded in continuous mode; and Vel-active source velocity surveys recorded.

Two different granular materials were used to simulate fault gouge: (1) Ottawa sand, a pure $>99 \%$ angular quartz sand obtained from the U.S. Silica Company, Ottawa, Illinois, and (2) precision soda lime glass spheres acquired from Mo-Sci Corporation, Rolla, Missouri. The initial particle size distribution and mean grain size of the two materials were comparable as were the compressive strengths (see Table 1 for experiment details). These particular materials were chosen to allow direct comparison to a substantial database of existing laboratory observations (e.g., Karner and Marone, 2001; Frye and Marone, 2002; Mair et al., 2002; Anthony and Marone, 2005).

\section{Ultrasonic Monitoring}

Acoustic waves generated during shear of granular layers were monitored by an array of piezoelectric transducers mounted on the steel (or granite) sample forcing blocks. Sensors were held in position using spring loaded sensor holders, and silicon grease was used as a couplant. The Panametrics V103 transducers used are compressional $(P$ wave) and single component with a dominant frequency of $\sim 1 \mathrm{MHz}$ and a diameter of $12 \mathrm{~mm}$. A full array consisted of 16 receivers and one transmitter distributed around the sheared layers (Fig. 1). Sensor positions were known to $\sim 1 \mathrm{~mm}$ accuracy and are reproducible on subsequent tests by using custom designed sensor holders. Signals were amplified near the source using $40 \mathrm{~dB}$ preamplifiers (PAC $1220 \mathrm{~A})$; then whole waveforms were conditioned and recorded using a multichannel ultrasonic acquisition system (ESG Hyperion Giga RAM recorder) capable of acquiring continuous and triggered signals on 16 channels at $10 \mathrm{MHz}$ and 16 bits of digital resolution (see Thompson et al. [2006] for details). This setup was also be used to monitor the ultrasonic velocity of the gouge during shear in a related study.

Triggered datasets are recorded when a waveform exceeds a predefined threshold level at a given sensor or set of sensors. Using a pretrigger ring buffer, the smaller amplitude first break of a waveform can be captured. Hence locations of events can be determined by comparing arrival times at an array of receivers. Triggered data are written to a hard drive immediately, and during this time no additional events can be acquired. Although this "dead time" is extremely short, it may result in an incomplete dataset at certain stages of loading when the AE rate is high. This limitation has recently been resolved with the advent of continuous $\mathrm{AE}$ monitoring, in which acquired waveform information is continuously streamed to RAM for temporary storage (Thompson et al., 2006). Using this approach, acoustic datasets have no dead time. After an experiment is complete, data are stored for subsequent processing. Both triggered and continuous datasets have been used to obtain the results presented in this article.

While acquiring waveform data, information on acoustic activity was extracted by automatically counting the number of triggered events recorded at each transducer in a given time interval. These data, from now on presented as AE per sec, form the basis of this manuscript. Importantly, however, we confirm our general results using an AE waveform record generated from continuous recording (see the Appendix). This allows us to test the following assumptions: (1) the majority of events recorded automatically are indeed bursttype "real" AE waveforms and not spurious noise, and (2) our main results are not artifacts of recording system saturation.

\section{Results}

\section{Friction Rate Dependence}

A typical friction versus displacement curve for granular material is shown in Figure 2. The plot highlights three initial "run-in" load cycles conducted at $40 \mathrm{MPa}$ normal stress (these cycles are used to generate a consistent starting state in the gouge layer for different experiments). Normal stress is reduced to $25 \mathrm{MPa}$ for the main stage of the experiment, which shows increasing friction to a peak value and then steady state sliding occurs at a value of $\mu \sim 0.6$. Perturbations about the steady state friction are due to imposed changes in the load point velocity (or imposed hold periods). The inset in Figure 2 shows a close-up of the friction response to step changes in loading velocity, highlighting the characteristic 


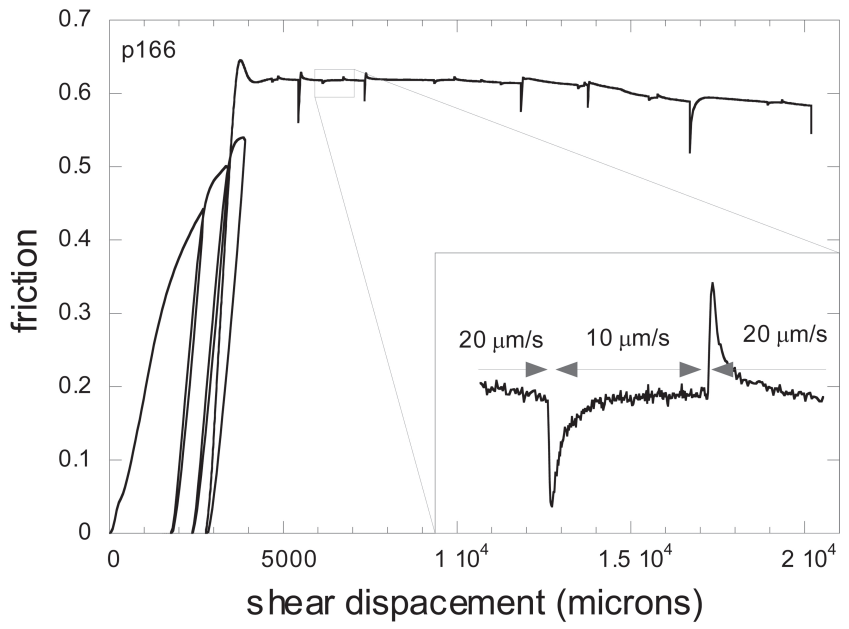

Figure 2. Typical friction shear displacement plot for sheared quartz sand showing a closeup of the frictional response to a step change in velocity, $V=20-10-20 \mu \mathrm{m} / \mathrm{sec}$ (inset).

direct effect (instantaneous resistance to the change) and e volution effect (decay back to a new steady state over a critical slip distance) commonly observed (e.g., Dieterich et al., 1981). Comparable tests were carried out for two simulated fault gouges: angular quartz sand and spherical glass beads (Table 1).

\section{Acoustic Emissions}

An AE whole waveform, typical of those recorded during shearing experiments, is presented in Figure 3. AE signals have a characteristic burst-type envelope with an obvious break of slope pinpointing the arrival at a given transducer. They require a short rise time to reach peak amplitude after which amplitude decays exponentially with time. Because we used compressional $P$-wave transducers, the $S$ wave arrival is generally suppressed.

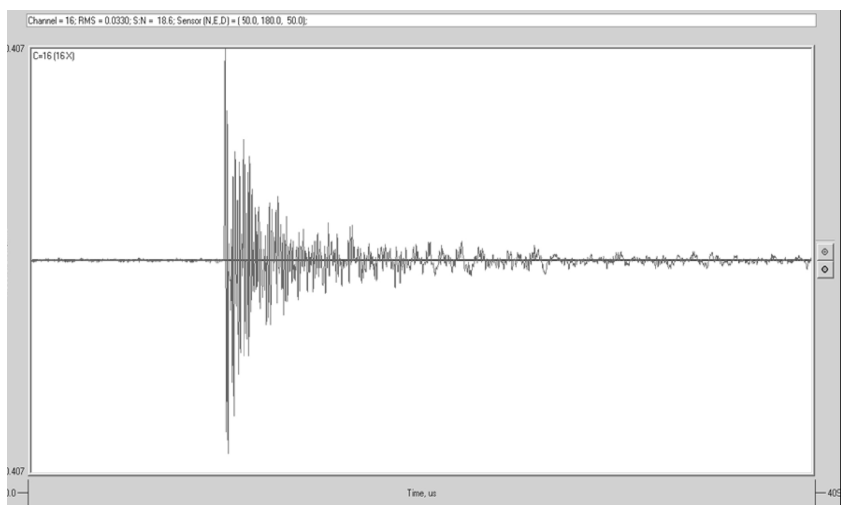

Figure 3. Triggered AE waveforms plotted as amplitude in $\mathrm{mV}$ versus time in $\mu \mathrm{sec}$. These waveforms were recorded during the latter stages of experiment P169, after $\sim \mathrm{mm}$ shear displacement at $10-15 \mathrm{MPa}$.
Figure 4 illustrates the response of friction and number of AE per sec to imposed step changes in loading velocity for two experiments (at different normal stresses). Friction exhibits the characteristic direct and evolution response to a step change in loading velocity consistent with previous observations (e.g., Mair and Marone, 1999). In all cases, an increase in loading velocity promotes an immediate and sustained increase in AE per sec. A decrease in velocity has the converse effect. For a given shear displacement range, changes in $\mathrm{AE}$ rate associated with velocity steps appear to be reversible (Fig. 4), indicating that the AE rate is not dominated by the preceding velocity condition.

\section{Rate Dependence of AE}

The mean number of AE per sec are plotted against shear displacement for two different experiments in Figure 5a and $b$. These data are calculated from AE per sec (similar to the data presented in Fig. 4) for each individual velocity segment. The positive influence of loading rate on AE activity described previously is clear. For a given shear displacement, AE activity for $V=30 \mu \mathrm{m} / \mathrm{sec}$ plots higher than $V=20 \mu \mathrm{m} / \mathrm{sec}$ data, which in turn plot higher than $V=10 \mu \mathrm{m} / \mathrm{sec}$ (Fig. 5a). For a given velocity, the AE event
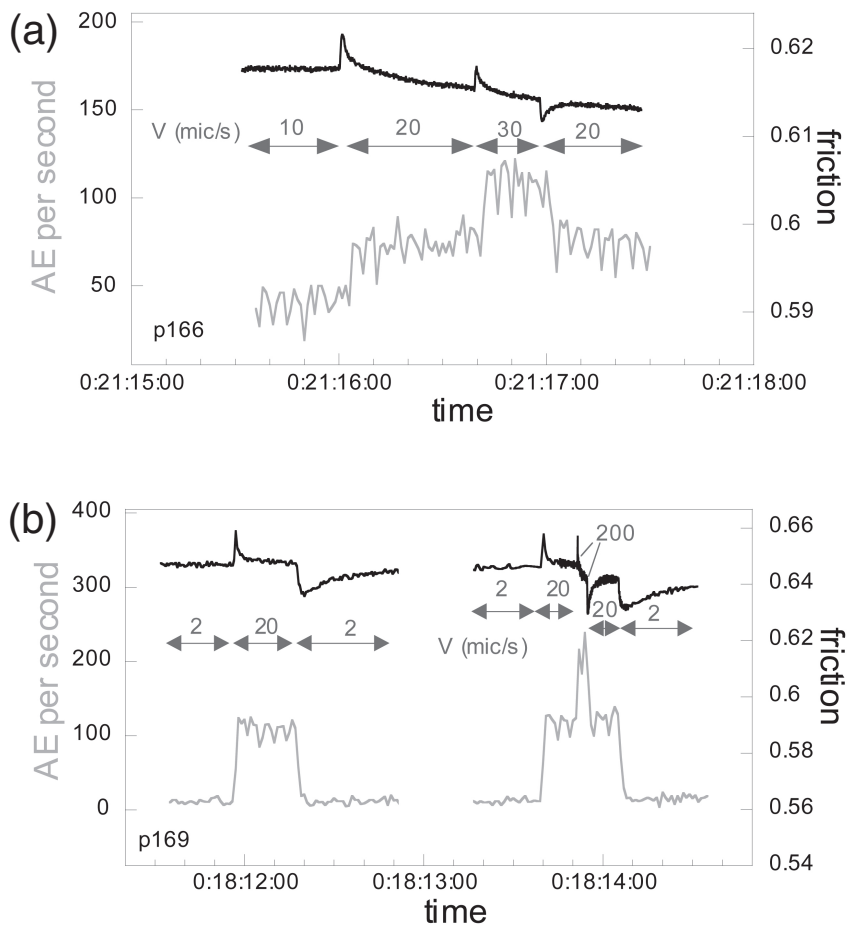

Figure 4. Friction (black lines) and number of AE per sec (gray lines) are plotted against time for a series of step changes in the load point velocity. Two experiments conducted at different normal stresses are shown. (a) P166 (25 MPa, $5 \mathrm{~mm}$ layers, F110) $V=10-20-30-20 \mu \mathrm{m} / \mathrm{sec}$ (at $10-12 \mathrm{~mm}$ shear displacement). (b) P169 (10/15 MPa, $1.5 \mathrm{~mm}$ layers, F110 on granite blocks) $V=2-20-2 \mu \mathrm{m} / \mathrm{sec} ; V=20-200-20 \mu \mathrm{m} / \mathrm{sec}$ (at 29-30 mm shear displacement). 


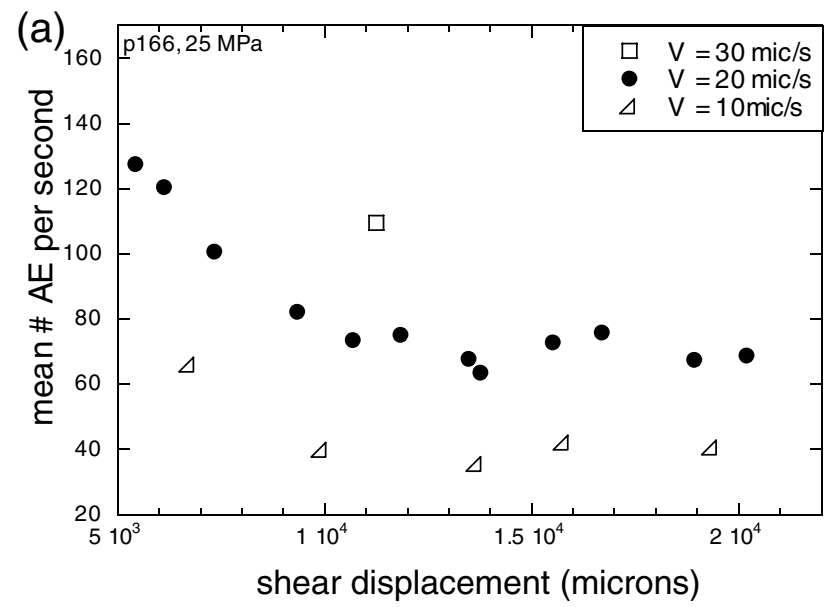

(b)

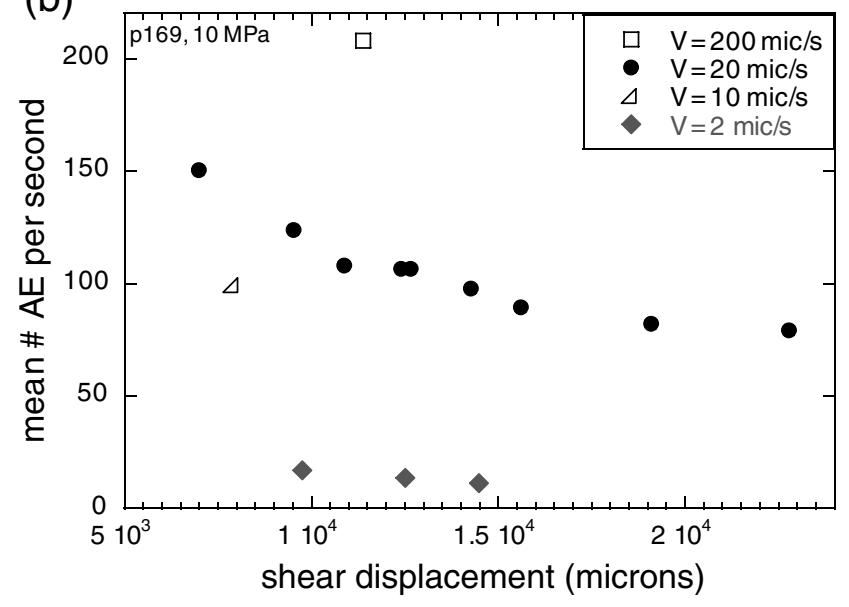

Figure 5. Mean AE per sec measured for constant velocity segments as a function of shear displacement for (a) P166 (25 MPa) and (b) P169 (10 MPa). Both are shown for shear displacement ranges $5-23 \mathrm{~mm}$.

rate decreased systematically but nonlinearly with accumulated slip; hence, a nonlinear displacement effect is apparent. This effect is initially strong but much reduced after $\sim 9 \mathrm{~mm}$ shear displacement. This is consistent with observations of rapid comminution and significant layer thinning during initial shear (Mair et al., 2002) that is shown to be enhanced at higher normal stress. For this reason, data subsequently presented in this article are for $>9 \mathrm{~mm}$ shear displacement, where this displacement effect (and layer thinning) is minimal and we can better investigate the rate dependence.

The influence of shearing velocity on AE rate is further explored in Figure 6. Mean AE per sec exhibits a positive systematic dependence on the logarithm of sliding velocity. This positive dependence on velocity is not entirely surprising because more slip is covered per unit time when shearing at higher velocities. To account for this, we normalize our data by load point velocity for each individual velocity segment. This effectively normalizes the data by slip, and we plot mean AE per $\mu \mathrm{m}$ slip in Figure 6b. If the process respon- sible for $\mathrm{AE}$ is rate independent, we would expect $\mathrm{AE}$ per unit slip to be constant for all sliding velocities. This is clearly not the case as the normalized AE rate (Fig. 6b) exhibits a systematic decrease with increasing log velocity, indicating a deficit of acoustic activity per unit slip at larger $V$.

Acoustic activity data from several experiments on angular quartz and spherical beads are summarized in Figure 7. These data confirm the general trends presented in Figure 6, indicating increased $\mathrm{AE}$ rate with increasing velocity and a decreasing AE per unit slip with the $\log$ of inverse $V$. Similar first-order trends are observed for both angular and spherical gouge material; however, some differences in response of the two materials are apparent. A gouge consisting of spherical grains shows a systematically larger number of $\mathrm{AE}$ per sec and a steeper decrease of AE per $\mu \mathrm{m}$ with increasing $V$ than an angular gouge. There is no first-order influence of applied normal stress on AE per sec or AE per $\mu \mathrm{m}$, but a systematic investigation of this has not been carried out.
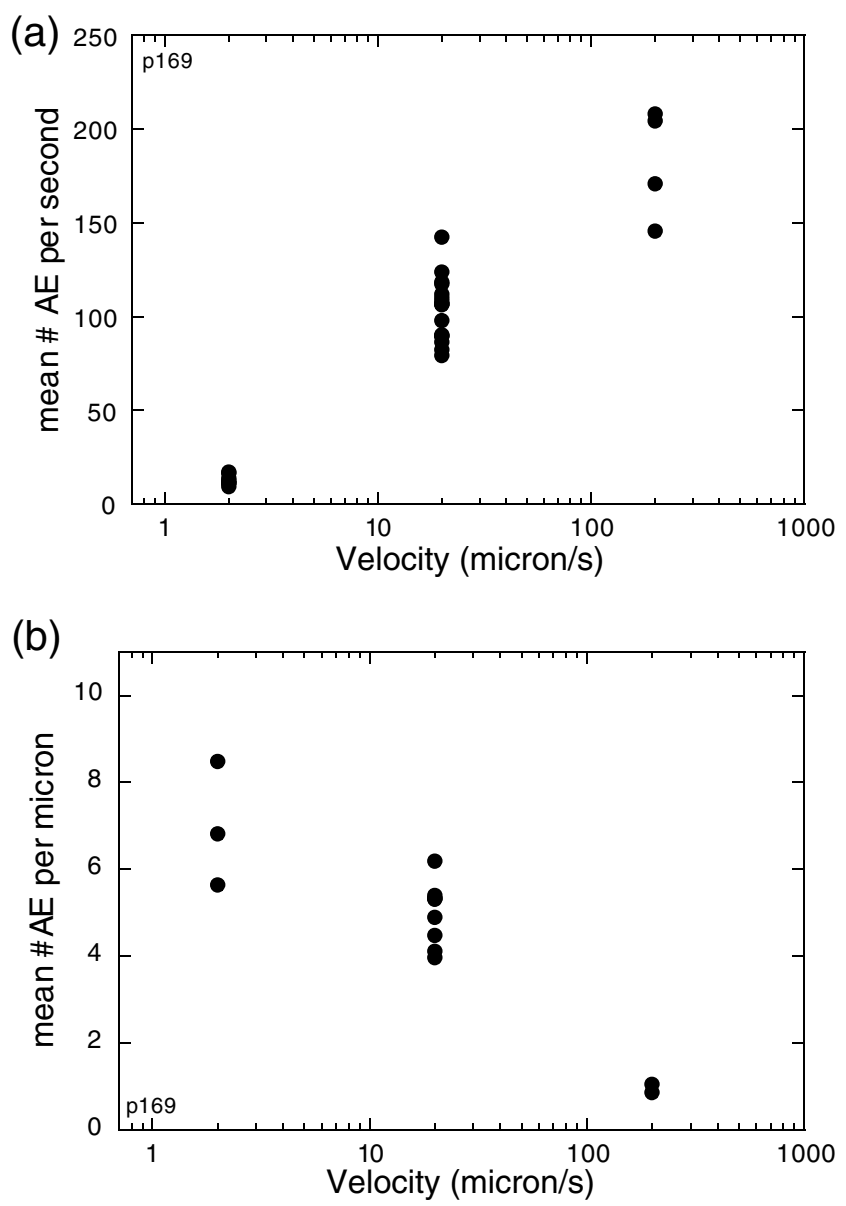

Figure 6. (a) Mean AE per sec and (b) mean AE per $\mu$ m plotted against the log of the load point velocity for a single experiment (P169). Data are for shear displacement 9-32 $\mathrm{mm}$ and indicate (a) a clear increase in $\mathrm{AE}$ per sec with a log increase in velocity and, in contrast, (b) a systematic decrease in AE per $\mu \mathrm{m}$ with the $\log$ of velocity. 

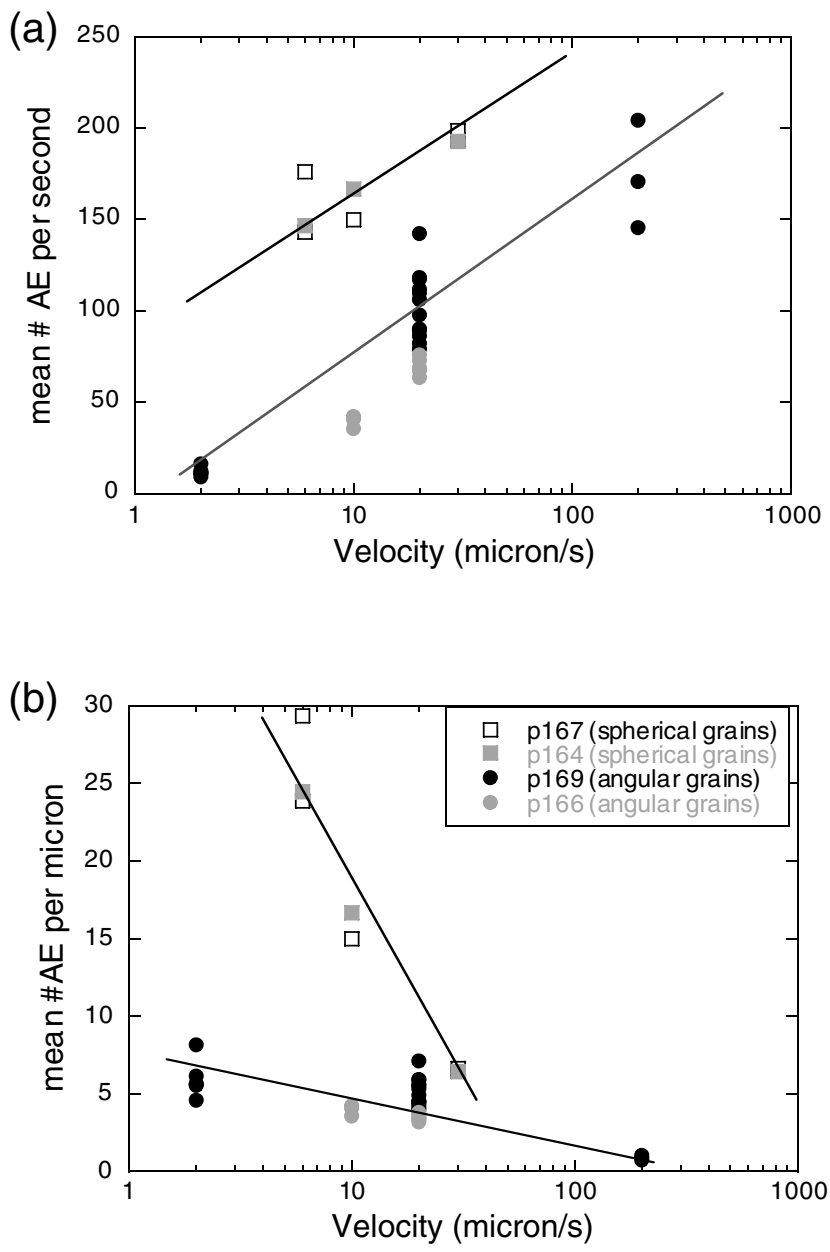

Figure 7. (a) Summary of mean AE per sec and (b) mean hits per $\mu \mathrm{m}$ plotted against the log of the load point velocity for several experiments. Spherical glass beads (P167 and P164) and angular quartz gouge (P166 and P169) indicate similar trends of increasing AE per sec with increasing velocity and decreasing AE per $\mu \mathrm{m}$ with increasing velocity. The absolute number of hits and rate of change varies between the two materials. The lines are hand drawn guides to highlight the main trends of the materials.

\section{Discussion}

Our investigation builds on recent work in which $\mathrm{AE}$ were measured for sliding on bare rock surfaces (Kato et al., 1994; Yabe, 2002; Yabe et al., 2003). To our knowledge there are no comparable studies on AE of a sheared simulated fault gouge, although gouge material was generated during the bare surface sliding experiments of Yabe and coworkers.

We show that for a given slip rate, mean AE activity evolves with accumulated slip, suggesting a sensitivity to gouge layer evolution. This is most likely related to comminution (i.e., grain size reduction associated with crushing and grinding) or possibly geometric layer thinning effects. Our observations show that the largest changes in $\mathrm{AE}$ activity occur at small amounts of slip. During initial shearing grain fracture would be expected to be intense (Sammis and Biegel, 1989; Marone and Scholz, 1989). We therefore infer that $\mathrm{AE}$ in the initial stages of the test are dominated by the fracture of intact grains with lesser contribution from frictional sliding. For the displacements $>9 \mathrm{~mm}$, we believe comminution rate is significantly reduced as slip becomes localized on internal shear bands (e.g., Mair and Marone, 2000), and therefore the dominant processes operating in the gouge layers are grain sliding (and grain rolling). We suggest then that AE generated after $\sim 9 \mathrm{~mm}$ slip (shear strain of $\sim 2$ ) are mainly due to contact junction rupture during frictional sliding along grain contacts. If this is the case, then it is reasonable to infer that the amount of AE activity (AE per unit slip) will be proportional to the real area of frictional contact between particles in motion (i.e., total area of contact junctions that are rupturing). We observe reduced AE activity (AE per unit slip) at increased slip rates, consistent with results of Yabe et al. (2003) for bare surfaces, suggesting, given our assumptions, that the frictional contact area is reduced at increased loading velocity. We find the same result for both types of gouge particles although they differ in the rates of decrease of AE. A sketch of this is presented in Figure 8. By corollary, this suggests that contact junction growth is time dependent, and the observation that $\mathrm{AE}$ rate varies with log sliding velocity suggests a log time dependence consistent with previous work (Dieterich, 1978).

We now consider the details of the contact area population evolution and possible insights we can gain from $\mathrm{AE}$ observations. Dieterich and Kilgore (1994) showed that the contact area could increase with time by either the growth of existing contacts or the addition of new ones. By corollary, it is reasonable that the converse statement also holds, that is, that the contact area may decrease by the shrinkage of existing contacts or the destruction of old contacts.

Previous AE studies on bare surface shearing experiments by Yabe et al. (2003) and Kato et al. (1994) have in-

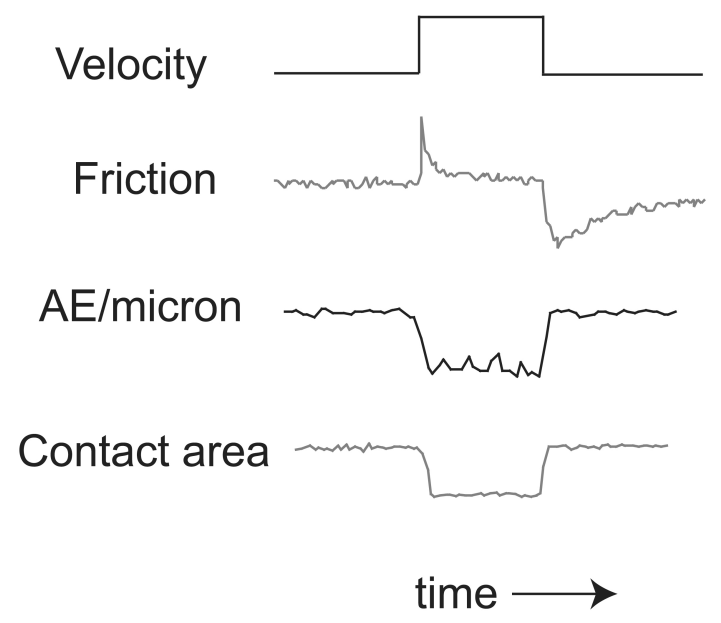

Figure 8. Schematic diagram illustrating the friction response, $\mathrm{AE}$ activity, and interpreted change in the contact area associated with step changes in loading velocity. 
terpreted the average source radius of $\mathrm{AE}$ events to be on the order 10 and $1.7 \mathrm{~mm}$, respectively. From direct observations of transparent materials (Dieterich and Kilgore, 1994) and the theory of contact between elastic rough surfaces (Yoshioka and Iwasa, 1996), it is suggested that spot size of asperity contacts depends on surface roughness and normal stress. On applying these results to their experiments, Yabe et al. (2003) estimate spot size of asperity contacts to be 10$100 \mu \mathrm{m}$. Because this asperity contact size is much smaller than the source size of AE events, they conclude that a single $\mathrm{AE}$ event cannot be due to the rupture of a single asperity and is instead caused by coherent rupture of many asperities (Kato et al., 1994; Yabe et al., 2003). From our current experiments on sheared gouges, it is not possible to directly determine the source size of each individual AE event. However, because our main $\mathrm{AE}$ results of reduced $\mathrm{AE}$ activity (i.e., AE per unit slip) at increased slip rates are consistent with those of bare surface experiments (Yabe et al., 2003), it is plausible that a similar scenario unfolds and that single AE events generated in sheared granular materials require the coherent rupture of many grain contacts.

Coherent rupture of asperity contacts along a fault surface, or part of a surface, is relatively easy to visualize, thanks to the efforts of Dieterich and Kilgore (1994) and of Yabe et al. (2003). When considering the geometric relations in sheared granular materials, however, it is not sufficient to consider deformation processes (in this case, slip or rupture of contact junctions) operating on a single surface along which slip is localized. One must consider the thousands of grain contacts and many degrees of freedom in a granular system that lead to a complicated contact population evolution. It has been proposed that a system of grain bridges or force chains preferentially carry load across sheared granular materials (Sammis et al., 1987; Sammis and Steacy, 1994; Cates et al., 1998). These grain bridges or force chains are transient features expressed by grains carrying higher than average contact forces. They persist only while they are favorably oriented. On breakdown, they will rapidly reform in optimal orientations producing a new (or adapted) population of high-force grain contacts. Recent work from laboratory experiments (Mair et al., 2002; Anthony and Marone, 2005) and 3D numerical modeling (Mair and Hazzard, 2007) suggests that the 3D spatial distribution of force chains and the manner in which they distribute load is highly sensitive to grain characteristics such as grain shape and grain size distribution. It is also envisaged (Mair et al., 2002; Mair and Hazzard, 2007) that the breakdown of networks of force chains will be sensitive to their 3D distribution and may be linked to second-order fluctuations in macroscopic stress.

We suggest that system spanning force chains exist (e.g., Cates et al., 1998; Mueth et al., 1998; Howell et al., 1999; Behringer, 2005) and are indeed the dominant load carrying network in our sheared gouge experiments. We propose that the breakdown of these force chains involves coherent slip on many grain contacts that together give rise to the individual $\mathrm{AE}$ events we monitor. The rate dependence of $\mathrm{AE}$ we observe can be interpreted by log time dependent growth of the grain contacts involved with individual force chains. These grain contacts will preferentially grow (with respect to other grain contacts not associated with the strong force chain network) due to the higher normal loads acting there. At low slip rates, individual grain contacts have time to grow (effectively heal) and therefore would be expected to have larger contact area leading to more AE activity during contact junction rupture. At higher slip rates, grain contacts are less mature, have little time to develop, and therefore have smaller contact areas and rupture to produce less $\mathrm{AE}$ activity. Differences in AE activity observed for spherical and angular gouges (Fig. 7) can be explained by considering the distinct style of 3D force chain networks expected to exist in the different granular materials. Mair et al. (2002) proposed that sheared spherical material (of relatively similar grain size distributions) would have discrete linear force chains producing high local normal stresses at grain contacts. In contrast, granular material composed of angular grains would be expected to have more diffuse force chain distributions with lower stresses at the individual contacts. Higher normal stresses could lead to larger contact junction areas, as shown by Dieterich and Kilgore (1994); hence, it is perceivable that spherical particles would have larger contact junction area and hence enhanced $\mathrm{AE}$ relative to angular gouge.

We have chosen to discuss our results in terms of force chains; however, it should be noted that gouge layer dilation may play a role in reducing the real area of contact junctions at high loading rates. The underlying mechanical mechanism for contact junction growth is generally perceived as thermally activated creep at asperity contacts where stresses are significantly enhanced. From our data we can offer no reasonable alternative.

Discerning the exact frictional contact area population and its evolution from AE activity in our current experiments is not feasible. Future work in this area would be valuable for testing ideas related to source size of individual AE events, frequency content of $\mathrm{AE}$, and $\mathrm{AE}$ source locations. A systematic analysis of friction parameters such as $D_{c}$ (critical slip distance) and gouge layer dilatancy for tests in which AE was monitored would be particularly useful, as would a systematic investigation of normal stress effects. A better understanding of 3D force chain geometries and force chain persistence as a function of slip and slip rate in sheared gouges is also required and may be attainable using new 3D fault gouge models (e.g., Abe and Mair, 2005).

\section{Conclusions}

We present new observations of acoustic emission activity produced during shearing of a simulated fault gouge. Our results indicate that the rate of acoustic activity (AE per $\mu \mathrm{m}$ of slip) is sensitive to accumulated slip and shows a systematic dependence on loading velocity. Our results 
qualitatively agree with previous experiments carried out on bare rock surfaces and support ideas that the frictional contact junction area is reduced at increased sliding velocity. We suggest that the evolution of force chain networks permits the coherent rupture of many grain contacts that leads to individual acoustic events and that distinct 3D force chain geometries account for subtle differences in the response of angular and spherical granular materials. Our results highlight a new way to image micromechanical processes operating in fault zones. The results are relevant to frictional mechanics and have application to earthquake physics and pave the way for further in-depth studies on acoustic imaging of sliding fault zones.

\section{Acknowledgments}

We thank Jen Anthony and Ben Thompson for assistance running friction experiments and processing the acoustic waveform data. Karen Mair was supported during this work by a Royal Society Dorothy Hodgkin Fellowship held at the University of Liverpool. This work was funded in part by National Science Foundation grants to Chris Marone: EAR-0196570, OCE0196462, and EAR-0337627. We acknowledge additional support from the Center for Physics of Geological Processes at the University of Oslo.

\section{References}

Abe, S., and K. Mair (2005). Grain fracture in 3D numerical simulations of granular shear, Geophys. Res. Lett. 32, L05305, doi 1029/ 2004GL022123.

Anthony, J. L., and C. Marone (2005). Influence of particle characteristics on granular friction, J. Geophys. Res. 110, no. B8, B08409.

Behringer, R. P. (2005). Contact force measurements and stress-induced anisotropy in granular materials, Nat. 435, 1079-1082.

Cates, M. E., J. P. Wittmer, J. P. Bouchaud, and P. Claudin (1998). Jamming, force chains, and fragile matter, Phys. Rev. Lett. 81, no. $9,1841-1844$.

Dieterich, J. H. (1978). Time dependent friction and mechanics of stick-slip, Pageoph 116, no. 4-5, 790-806.

Dieterich, J. H. (1979). Modeling rock friction. 1. Experimental results and constitutive equations, J. Geophys. Res. 84, 2161-2168.

Dieterich, J. H. (1981). Constitutive properties of faults with simulated gouge, in Mechanical Behaviour of Crustal Rocks, N. L. Carter, J. M. Logan and D. W. Stearns (Editors), American Geophysical Monograph 24.

Dieterich, J. H., and B. D. Kilgore (1994). Direct observations of frictional contacts - new insights for state-dependent properties, Pageoph 143, no. 1-3 283-302.

Frye, K. M., and C. Marone (2002). The effect of particle dimensionality on granular friction in laboratory shear zones, Geophys. Res. Lett. 29, no. 19, 1916.

Howell, D., R. Behringer, and C. Veje (1999). Stress fluctuations in a couette experiment: a continuous transition, Phys. Rev. Lett. 82, no. 26, 5241-5244.

Karner, S. L., and C. Marone (1998). The effect of shear load on frictional healing in simulated fault gouge, Geophys. Res. Lett. 25, no. 24, 45614564.

Karner, S. L., and C. Marone (2001). Frictional restrengthening in simulated fault gouge: effect of shear load perturbations, J. Geophys. Res. 106, no. B9, 19,319-19,337.

Kato, N., K. Yamamato, and T. Hirasawa (1994). Microfracture processes in the breakdown zone during dynamic shear rupture inferred from laboratory observation of near-fault high-frequency strong motion, Pageoph 142, 713-734.
Lockner, D. (1993). The role of acoustic emissions in the study of rock fracture, Int. J. Rock Mech. Miner. Sci. Geomech. Abstr. 30, 883-899.

Mair, K., and J. F. Hazzard (2007). Nature of stress accommodation in sheared granular material: insights from 3D numerical modeling, Earth Planet. Sci. Lett. 259, 469-485, doi 10.1016/j.eps1.2007.05.006.

Mair, K., and C. Marone (1999). Friction of simulated fault gouge for a wide range of velocities and normal stresses, J. Geophys. Res. 104, 28,89928,914 .

Mair, K., and C. Marone (2000). Shear heating in granular layers, Pure Appl. Geophys. 157, 1847-1866.

Mair, K., K. M. Frye, and C. Marone (2002). Influence of grain characteristics on the friction of granular shear zones, J. Geophys. Res. 107, no. 10, 2219, doi 10.1029/2001JB000516.

Marone, C. (1998). Laboratory-derived friction laws and their application to seismic faulting, Ann. Rev. Earth Planet. Sci. 26, 643-696.

Marone, C., and C. Scholz (1989). Particle-size distribution and microstructures within simulated fault gouge, J. Struct. Geol. 11, no. 7, 799-814.

Mueth, D. M., H. M. Jaeger, and S. R. Nagel (1998). Force distribution in a granular medium, Phys. Rev. E 57, no. 3, 3164-3169.

Paterson, M. S., and T.-F. Wong (2005). Experimental Rock DeformationThe Brittle Field, Second Ed., Springer, New York.

Sammis, C. G., and R. L. Biegel (1989). Fractals, fault-gouge and friction, Pageoph 131, no. 1-2, 255-271.

Sammis, C. G., and S. J. Steacy (1994). The micromechanics of friction in a granular layer, Pageoph 142, 777-794.

Sammis, C., G. King, and R. Biegel (1987). The kinematics of gouge deformation, Pageoph 125, no. 5, 777-812.

Sammonds, P., and M. Ohnaka (1998). Evolution of microseismicity during frictional sliding, Geophys. Res. Lett. 25, no. 5, 699-702.

Scholz, C. H. (1998). Earthquakes and friction laws, Nat. 391, 37-42.

Thompson, B. D., R. P. Young, and D. A. Lockner (2006). Fracture in Westerly granite under $\mathrm{AE}$ feedback and constant strain rate loading: nucleation, quasi-static propagation, and the transition to unstable fracture propagation, Pageoph 163, no. 5-6, 995-1019.

Weeks, J., D. Lockner, and J. Byerlee (1978). Changes in b-values during movement on cut surfaces in granite, Bull. Seismol. Soc. Am. 68, 333341.

Yabe, Y. (2002). Rate dependence of AE activity during frictional sliding, Geophys. Res. Lett. 29, no. 10, 1388.

Yabe, Y., N. Kato, K. Yamamoto, and T. Hirasawa (2003). Effect of sliding rate on the activity of acoustic emission during stable sliding, Pageoph 160, $1163-1189$.

Yoshioka, N., and K. Iwasa (1996). The characteristic displacement in rate and state dependent friction from a micromechanical point of view, Pageoph 147, 433-453.

\section{Appendix}

We conducted two validity tests to determine (1) whether triggered AE measured automatically are directly correlated to burst-type ultrasonic events and (2) if the majority of acoustic activity is actually captured, that is, to ensure the system is not saturated and undercounting AE at high load point velocities (because this could produce an apparent deficit of AE at high $V$ ). Both validity tests utilized continuous waveform data recorded during a short section of a test using the Giga RAM recorder. These data were processed to generate a synthetic triggered dataset-equivalent to that recorded automatically by a traditional ultrasonic system but with no dead time.

1. Several hundred waveforms from these synthetic triggered events were analyzed by hand to determine the proportion of triggered events interpreted to be real burst- 


\section{Table A1}

AE Activity Data Obtained from Synthetic Triggered Dataset for Experiment P167

\begin{tabular}{ccc}
\hline P167 & $V=6 \mu \mathrm{m} / \mathrm{sec}$ & $V=30 \mu \mathrm{m} / \mathrm{sec}$ \\
\hline Mean number AE per sec & 54.6 & 130.7 \\
Mean number AE per $\mu \mathrm{m}$ & 9.1 & 4.2 \\
\hline
\end{tabular}

type signals (where the onset of the event and a short rise time to peak amplitude could be clearly identified) versus those interpreted as being due to coda ringing (from previous large events) or other spurious noise. Over $75 \%$ of events showed burst-type signals (similar to Fig. 3), indicating that at least $75 \%$ are associated with real acoustic events. This confirms that the triggered waveform data are dominated by real acoustic events and not spurious noise.

2. From the triggered dataset we also summed the number of $\mathrm{AE}$ hits per sec to generate an independent record of the number of $\mathrm{AE}$ activity. Unlike the auto-AE per sec counter, this $\mathrm{AE}$ per sec data has no saturation limit per se (because records are continuous) and will miss events only if more that one event occurs during the time for a single waveform trace $(0.4 \mathrm{msec})$. AE per sec data generated by this method agree well with the trends shown in the relevant autohit data, that is, mean $\mathrm{AE}$ per sec increases with increasing $V$ and mean AE per $\mu$ m decreases with increasing $V$ (Table A1). The actual number of AE per sec differs between synthetic and real datasets because the threshold levels and the time window (effectively the event duration) are different in the autotriggered and processed continuous Giga data.

\author{
Physics of Geological Processes \\ University of Oslo \\ P.O. Box 1048, Blindern \\ Oslo, 0460 Norway \\ karen.mair@fys.uio.no \\ (K.M.) \\ Department of Geosciences \\ Pennsylvania State University \\ 536 Deike Building \\ University Park, PA 16802 \\ cjm@geosc.psu.edu \\ (C.M.) \\ Lassonde Institute \\ University of Toronto \\ 170 College Street \\ Toronto, Ontario, M5S 3E3 Canada \\ paul.young@utoronto.ca
}

(R.P.Y.)

Manuscript received 16 November 2006 